\title{
Improving Implantation Rate in 2nd ICSI Cycle through Ovarian Stimulation with FSH and LH in GNRH Antagonist Regimen
}

\section{Melhorando a taxa de implantação no $2^{\circ}$ ciclo de ICSI através do estímulo ovariano com FSH e $\mathrm{LH}$ no regime com antagonista do GnRH}

\author{
Amanda Souza Setti ${ }^{1}$ Daniela Paes de Almeida Ferreira Braga ${ }^{10}$ Assumpto laconelli Júnior ${ }^{1}$ () \\ Edson Borges Júnior ${ }^{10}$ \\ ${ }^{1}$ Fertility Medical Group, São Paulo, SP, Brazil \\ Address for correspondence Amanda Setti, M.Sc., Av. Brigadeiro Luis \\ Antonio, 4545, 01401-002, São Paulo, SP, Brazil \\ Rev Bras Ginecol Obstet 2021;43(10):749-758. \\ (e-mail: amanda@sapientiae.org.br).
}

\begin{abstract}
Keywords

- follicle-stimulating hormone

- intracytoplasmic sperm injection implantation

- luteinizing hormone

- ovarian stimulation

Objective To investigate whether patients with a previous recombinant follicle stimulating hormone ( $\mathrm{rFSH}$ )-stimulated cycle would have improved outcomes with $\mathrm{rFSH}+$ recombinant luteinizing hormone $(\mathrm{rLH})$ stimulation in the following cycle.

Methods For the present retrospective case-control study, 228 cycles performed in 114 patients undergoing intracytoplasmic sperm injection (ICSI) between 2015 and 2018 in an in vitro fertilization (IVF) center were evaluated. Controlled ovarian stimulation (COS) was achieved with rFSH (Gonal-f, Serono, Geneva, Switzerland) in the first ICSI cycle (rFSH group), and with rFSH and rLH (Pergoveris, Merck Serono S.p.A, Bari, Italy) in the second cycle ( $\mathrm{rFSH}+\mathrm{rLH}$ group). The ICSI outcomes were compared among the groups.

Results Higher estradiol levels, oocyte yield, day-3 high-quality embryos rate and implantation rate, and a lower miscarriage rate were observed in the rFSH + rLH group compared with the rFSH group. In patients $<35$ years old, the implantation rate was higher in the $\mathrm{rFSH}+\mathrm{rLH}$ group compared with the rFSH group. In patients $\geq 35$ years old, higher estradiol levels, oocyte yield, day-3 high-quality embryos rate, and implantation rate were observed in the $\mathrm{rFSH}+\mathrm{rLH}$ group. In patients with $\leq 4$ retrieved oocytes, oocyte yield, mature oocytes rate, normal cleavage speed, implantation rate, and miscarriage rate were improved in the $\mathrm{rFSH}+\mathrm{rLH}$ group. In patients with $\geq 5$ retrieved oocytes, higher estradiol levels, oocyte yield, and implantation rate were observed in the rFSH + rLH group.

Conclusion Ovarian stimulation with luteinizing hormone (LH) supplementation results in higher implantation rates, independent of maternal age and response to $\mathrm{COS}$ when compared with previous cycles stimulated with rFSH only. Improvements were also observed for ICSI outcomes and miscarriage after stratification by age and retrieved oocytes.
\end{abstract}

received

December 1, 2020 accepted after revision August 26, 2021
DOI https://doi.org/ 10.1055/s-0041-1736306. ISSN 0100-7203. (c) 2021. Federação Brasileira de Ginecologia e Obstetrícia. All rights reserved.

This is an open access article published by Thieme under the terms of the Creative Commons Attribution License, permitting unrestricted use, distribution, and reproduction so long as the original work is properly cited. (https://creativecommons.org/licenses/by/4.0/)

Thieme Revinter Publicações Ltda., Rua do Matoso 170, Rio de Janeiro, RJ, CEP 20270-135, Brazil 


\section{Resumo}

\author{
Palavras-chave \\ - hormônio folículo- \\ estimulante \\ - microinjeção \\ intracitoplasmática \\ de espermatozoides \\ - hormônio \\ luteinizante \\ - estímulo ovariano
}

Objetivo: Investigar se há algum efeito da suplementação com hormônio luteinizante (LH, na sigla em inglês) no regime com antagonista do hormônio liberador de gonadotropina ( $\mathrm{GnRH}$, na sigla em inglês) sobre os resultados dos ciclos consecutivos de injeção intracitoplasmática de espermatozoides (ICSI, na sigla em inglês).

Métodos Para o presente estudo retrospectivo de caso-controle, foram avaliados 228 ciclos de microinjeção intracitoplasmática de espermatozoides (ICSI, na sigla em inglês) realizados em 114 pacientes entre 2015 e 2018 em um centro privado de fertilização in vitro (FIV) afiliado a uma universidade. O estímulo ovariano controlado (EOC) foi feito com hormônio folículo- estimulante recombinante ( $r F S H$, na sigla em inglês) (Gonal-f, Serono, Genebra, Suíça) no primeiro ciclo de ICSI (grupo rFSH), e com rFSH e rLH (Pergoveris, Merck Serono S.p.A, Bari, Itália) no segundo ciclo (grupo $\mathrm{rFSH}+\mathrm{rLH}$ ). Os desfechos dos ciclos de ICSI foram comparados entre os grupos.

Resultados Níveis mais elevados de estradiol, de recuperação oocitária, taxa de embriões de alta qualidade no $3^{\circ}$ dia e taxa de implantação, e menor taxa de aborto foram observados no grupo rFSH + rLH. Em pacientes $<35$ anos, a taxa de implantação foi maior no grupo rFSH + rLH em comparação com o grupo rFSH. Em pacientes com $\geq$ 35 anos, maiores níveis de estradiol, recuperação oocitária, a taxa de embriões de alta qualidade no $3^{\circ}$ dia e a taxa de implantação foram observados no grupo rFSH + rLH. Em pacientes com baixa resposta ao EOC ( $\leq 4$ oócitos recuperados), a recuperação oocitária, a taxa de oócitos maduros, a taxa de velocidade normal de clivagem, a taxa de implantação e a taxa de aborto foram melhoradas no grupo rFSH $+\mathrm{rLH}$. Em pacientes com resposta normal ao EOC ( $\geq 5$ oócitos recuperados), níveis mais elevados de estradiol, recuperação oocitária e taxa de implantação foram observados no grupo $\mathrm{rFSH}+\mathrm{rLH}$.

Conclusão A estimulação ovariana com suplementação de LH resultou em taxas de implantação mais altas, independentemente da idade materna e da resposta ao EOC, em comparação com os ciclos anteriores estimulados apenas com rFSH. Melhorias também foram observadas nos resultados da ICSI e na taxa de aborto quando as pacientes foram estratificadas por idade e número de oócitos recuperados.

\section{Introduction}

For assisted reproductive technology (ART), gonadotropinreleasing hormone $(\mathrm{GnRH})$ and gonadotropins are routinely administered for controlled ovarian stimulation (COS). For that, recombinant follicle stimulating hormone ( $\mathrm{rFSH}$ or follitropin alfa) and recombinant luteinizing hormone (rLH or lutropin alfa) are the key hormonal stimulus, which can be used individually or in combination. Follicle stimulating hormone and LH play distinct but complementary roles in follicle regulation, leading to synergistic actions in stimulating the recruitment and development of ovarian follicles, increasing follicle estradiol secretion, and completing oocyte maturation and subsequent ovulation. ${ }^{1}$

Although ovarian stimulation is essential for the success of ART, it is also known to reduce endogenous FSH and LH releases. ${ }^{2}$ Particularly, GnRH antagonists induce a profound pituitary supression, avoiding premature LH surge. Consequently, recruited follicles are radically deprived of $\mathrm{LH}$ sustenance. Exogenous FSH stimulation will support follicular development in most patients undergoing ART; however, up to $12 \%$ of the patients will not respond to FSH stimulation alone, which can happen due to the absence of $\mathrm{LH}^{3}$ For this subpopulation of patients, there is evidence that LH supplementation to FSH administration could be advantageous. ${ }^{3-10}$

In fact, rLH was originally commercialized to supplement follitropin alfa administration for specific patients, especially those presenting with severe LH and FSH deficiency, namely hypogonadotropic hypogonadism. More recently, new products were developed in a fixed combination of 2:1 (150 IU of $\mathrm{rFSH}$ and $75 \mathrm{IU}$ of $\mathrm{rLH}$ ), under the presupposition that this is the optimal FSH:LH ratio for the purpose of stimulating follicular development. Then, it was agreed that patients (i) with previous poor response to ovarian stimulation, (ii) inadequate ovarian response in the treatment in progress, (iii) aged $\geq 35$ years old could also benefit from $\mathrm{LH}$ supplementation. ${ }^{10}$

A recent meta-analysis that included 36 randomized controlled trials investigated the effectiveness of rLH combined with rFSH for COS compared with rFSH alone in 8,125 women undergoing ART. Moderate quality evidence that the use of rLH combined with rFSH may lead to more ongoing 
pregnancies than rFSH alone was observed. No evidence of a difference between the two regimens was observed in terms of live birth rate. The authors concluded that the evidence was insufficient to encourage or discourage stimulation regimens that include $\mathrm{rLH}$ combined with $\mathrm{rFSH}$ in ART. ${ }^{11}$

To date, there is no evidence that ovarian simulation with rLH improves ART outcomes in an unselected subpopulation. In addition, there is only one previous study that investigated cycles in which patients acted as their own controls. The objective of the present study was to investigate whether patients with a previous rFSH-stimulated cycle would have improved outcomes with $\mathrm{rFSH}+\mathrm{rLH}$ stimulation in the following cycle.

\section{Methods}

\section{Experimental Design, Patients, and Inclusion and Exclusion Criteria}

The present case-control within-subject study included data obtained via chart review of 228 cycles performed in 114 patients undergoing ICSI between 2015 and 2018 in a private university-affiliated IVF center. For all patients, rFSH (Gonalf, Serono, Geneva, Switzerland) was used for COS in the first ICSI cycle (rFSH group, $n=114$ ), followed by ovarian stimulation with rFSH and rLH (Pergoveris, Merck Serono S.p.A, Bari, Italy) in the next cycle (rFSH + rLH group, $n=114)$. Pituitary suppression was achieved with GnRH antagonist (cetrorelix acetate, Cetrotide; Merck KGaA, Darmstadt, Germany) in both groups.

The inclusion criteria were: couples with primary infertility undergoing their first rFSH-stimulated ICSI cycle, with intended fresh embryo transfer on day 5 of embryo development, who underwent a second $\mathrm{rFSH}+\mathrm{rLH}$ stimulated ICSI cycle, also intending fresh embryo transfer on day 5 of embryo development.

The exclusion criteria were as follows: Female patients undergoing ICSI cycles with vitrified/thawed or donated oocytes, surgical sperm retrieval, cryopreserved sperm, and vitrified/thawed embryo transfer.

Ovarian response to COS and ICSI outcomes were compared between the groups.

All patients signed a written informed consent form. The present study was approved by the local Institutional Review Board.

\section{Controlled Ovarian Stimulation}

For the first ICSI cycle of the patients, COS was started on the $3^{\text {rd }}$ day of the cycle, with the administration of daily doses of r-FSH. For the second ICSI cycle, on the $3^{\text {rd }}$ day of the cycle, $\mathrm{COS}$ was started with the administration of r-FSH $+\mathrm{r}-\mathrm{LH}$.

The following steps were the same for both the first and the second ICSI cycles. When at $\geq 1$ follicle $\geq 14 \mathrm{~mm}$ was visualized, pituitary blockage was performed using GnRHa. When $\geq 3$ follicles attained a mean diameter $\geq 17 \mathrm{~mm}$ and adequate serum estradiol levels were observed, final follicular maturation was triggered by the administration of $250 \mu \mathrm{g}$ of r-hCG (Ovidrel, Merck KGaA, Geneva, Switzerland) or $\mathrm{GnRH}$ agonist (triptorelin $0.2 \mathrm{mg}$, Gonapeptyl; Ferring
$\mathrm{GmbH}$, Kiel, Germany; or leuprolide acetate 2.0mg, Lupron Kit, Abbott S.A Societé Française des Laboratoires, Paris, France). Oocyte retrieval was performed 35 hours later.

\section{Intracytoplasmic Sperm Injection and Embryo Quality and Transfer}

Intracytoplasmic sperm injection was performed according to Palermo et al. ${ }^{12}$ Embryos were cultured in $50-\mu \mathrm{L}$ drops culture medium (Global, LifeGlobal, Guilford, USA) covered with paraffin oil, in a humidified atmosphere under $6 \% \mathrm{CO}_{2}$, at $37^{\circ} \mathrm{C}$, for 5 days. The embryos were morphologically evaluated on days 3 and 5 of development. On day 5,1 to 2 embryos were transferred per patient, depending on maternal age and embryo quality, using a soft catheter with transabdominal ultrasound guidance.

\section{Clinical Follow-Up}

A serum pregnancy test was performed 10 days after embryo transfer. Women with a positive $\beta$ human Chorionic Gonadotropin ( $\beta \mathrm{hCG}$ ) test underwent a transvaginal ultrasound scan after 2 weeks. Clinical pregnancy was confirmed when at least one intrauterine gestational sac with fetal heartbeat was detected. Implantation rate was calculated per transferred embryos. Clinical pregnancy rates were calculated per embryo transfer. Miscarriage was defined as pregnancy loss before 20 weeks of gestation.

\section{Data Analysis and Statistics}

The sample size calculation suggested that 200 cycles would be enough to demonstrate a $20 \%$ effect with $80 \%$ power and $5 \%$ significance level considering as primary outcome the implantation rate.

In the first analysis, response to COS, and the outcomes of ICSI were compared between the rFSH and rFSH + rLH groups ( $n=228$ ), using generalized linear models followed by the Bonferroni post hoc test. Then, data were stratified according to female age ( $<35$ years old, $n=50$, and $\geq 35$ years old, $n=178$ ) and response to $\operatorname{COS}$ (poor response: $\leq 4$ retrieved oocytes, $n=102$, and normal response: $\geq 5$ retrieved oocytes, $n=126$ ), and were reanalyzed as mentioned above. In all models, female age, body mass index (BMI) and total FSH dose were included as covariates. No patient has shifted age categories from the $1^{\text {st }}$ to the $2^{\text {nd }}$ ICSI cycle. Patients that became pregnant in the $1^{\text {st }}$ ICSI cycle and returned for a $2^{\text {nd }}$ cycle desiring another child were not excluded from the analysis, to avoid bias.

Data are expressed as mean \pm standard error for continuous variables or as percentages for dichotomous variables, and p-values. P-value was significant at $5 \%$ level $(<0.05)$. The analysis was performed using IBM SPSS Statistics for Windows, version 21 (IBM Corp., Armonk, NY, USA).

\section{Results}

All patients completed the follow-up (20 weeks of gestation), and there were no data missing regarding the reported variables. Higher estradiol levels $(1151.73 \pm 194.34 \mathrm{pg} / \mathrm{mL}$ versus $1909.11 \pm 194.34 \mathrm{pg} / \mathrm{mL}, p=0.006)$, oocyte yield 
Table 1 Descriptive analysis of demographics, response to COS and laboratorial ICSI outcomes of patients in repeated cycles $(n=228)$

\begin{tabular}{|c|c|c|c|}
\hline Variables & rFSH group $(n=114)$ & rFSH + rLH group $(n=114)$ & p-value \\
\hline Female age & $37.19 \pm 0.35$ & $37.89 \pm 0.35$ & 0.160 \\
\hline Male age & $39.23 \pm 0.63$ & $39.96 \pm 0.64$ & 0.416 \\
\hline BMI & $24.88 \pm 0.42$ & $24.68 \pm 0.42$ & 0.740 \\
\hline FSH dose (IU) & $2826.92 \pm 199.67$ & $2693.64 \pm 198.79$ & 0.636 \\
\hline LH dose (IU) & 0.0 & $1346.82 \pm 34.50$ & NA \\
\hline Estradiol level (pg/mL) & $1151.73 \pm 194.34$ & $1909.11 \pm 194.34$ & 0.006 \\
\hline Cycles triggered with GnRHa & $9 / 114(7.9)$ & $10 / 114(8.8)$ & 0.811 \\
\hline Follicles (n) & $9.99 \pm 0.70$ & $10.38 \pm 0.70$ & 0.695 \\
\hline Retrieved oocytes (n) & $6.37 \pm 0.49$ & $7.30 \pm 0.49$ & 0.185 \\
\hline Oocyte yield (\%) & $63.41 \pm 2.24$ & $69.78 \pm 2.24$ & 0.045 \\
\hline MII oocyte rate (\%) & $67.72 \pm 2.53$ & $71.48 \pm 2.52$ & 0.293 \\
\hline Fertilization rate (\%) & $77.33 \pm 2.41$ & $73.02 \pm 2.37$ & 0.202 \\
\hline Normal cleavage speed rate (\%) & $67.16 \pm 3.16$ & $73.07 \pm 3.11$ & 0.182 \\
\hline D3 high - quality embryos rate (\%) & $34.13 \pm 4.37$ & $47.71 \pm 4.40$ & 0.029 \\
\hline Blastocyst development rate (\%) & $36.72 \pm 6.68$ & $42.68 \pm 5.73$ & 0.499 \\
\hline Frozen embryos (n) & $2.21 \pm 0.61$ & $3.05 \pm 0.57$ & 0.308 \\
\hline Endometrial thickness (mm) & $10.32 \pm 0.27$ & $10.71 \pm 0.25$ & 0.288 \\
\hline Embryos transferred (n) & $2.08 \pm 0.09$ & $2.04 \pm 0.09$ & 0.759 \\
\hline Cycles with embryo transfer (\%) & $70 / 114(61.4)$ & $69 / 114(60.5)$ & 0.892 \\
\hline Implantation rate (\%) & $18.57 \pm 0.52$ & $26.47 \pm 0.62$ & $<0.001$ \\
\hline Pregnancy rate (\%) & $15 / 70(21.4)$ & $20 / 69(29.0)$ & 0.303 \\
\hline Miscarriage rate (\%) & $5 / 15(33.0)$ & $1 / 20(5.0)$ & 0.031 \\
\hline OHSS rate (\%) & $3 / 114(2.6)$ & $6 / 114(5.3)$ & 0.308 \\
\hline
\end{tabular}

Abbreviations: BMI, body mass index; COS, controlled ovarian stimulation; D3, day 3 of embryo development; ICSI, intracytoplasmic sperm injection; $\mathrm{IU}$, international unit; MII, metaphase II; NA, not applicable; OHSS, ovarian hyper stimulation syndrome; rFSH, recombinant follicle stimulating hormone; rLH, recombinant luteinizing hormone.

Note: values are mean \pm standard error, unless otherwise noted.

(63.41 versus $69.78 \%, p=0.045)$, day-3 high-quality embryos rate ( 34.13 versus $47.71 \%, p=0.029$ ) and implantation rate ( 18.57 versus $26.47 \%, p<0.001$ ), and lower miscarriage rate (33.0 versus $5.0, p=0.031$ ) were observed in the $\mathrm{rFSH}+\mathrm{rLH}$ group compared with the rFSH group ( - Table 1 ).

In patients $<35$ years old, the implantation rate was significantly higher in the $\mathrm{rFSH}+\mathrm{rLH}$ group compared with the rFSH group (21.43 versus $38.46 \%, p<0.001$ ) (-Table 2).

In patients aged $\geq 35$ years old, higher estradiol levels $(1161.80 \pm 215.94 \mathrm{pg} / \mathrm{mL}$ versus $1966.55 \pm 220.13 \mathrm{pg} / \mathrm{mL}$, $p=0.009$ ), oocyte yield (61.28\%versus $68.62 \%, p=0.038$ ), day-3 high-quality embryos rate (32.01 versus $48.81 \%$, $p=0.013)$, and implantation rate (17.35 versus $23.64 \%$, $p<0.001$ ) were observed in the $\mathrm{rFSH}+\mathrm{rLH}$ group compared with the rFSH group ( - Table 3 ).

In patients with poor response to $\operatorname{COS}(\leq 4$ retrieved oocytes), oocyte yield (56.82 versus $63.29 \%, p=0.001$ ), mature oocytes rate $(69.87$ versus $78+12 \%, p<0.001)$, normal cleavage speed ( 62.5 versus $75.83 \%, p<0.001$ ), implantation rate $(10.00$ versus $20.45 \%, p<0.001)$ and miscarriage rate
(100 versus $0.00 \%, p<0.001$ ) were improved in the $\mathrm{rFSH}+$ rLH group compared with the rFSH group ( - Table 4 ).

In patients with normal response to $\operatorname{COS}(\geq 5$ retrieved oocytes), higher estradiol levels $(1725.74 \pm 303.65 \mathrm{pg} / \mathrm{mL}$ versus $2788.37 \pm 281.12 \mathrm{pg} / \mathrm{mL}, p=0.010)$, oocyte yield (75.37 versus $82.69 \%, p=0.006$ ), and implantation rate ( 23.33 versus $29.35 \%, p<0.001$ ) were observed in the $\mathrm{rFSH}$ + rLH group compared with the rFSH group ( - Table 5 ).

\section{Discussion}

In the present study, we observed that COS with $\mathrm{rFSH}+\mathrm{rLH}$ resulted in higher estradiol levels, oocyte yield, day-3 highquality embryos rate and implantation rate, and lower miscarriage rate compared with COS with rFSH only. The only previous study that has investigated the effect of adding rLH to stimulation in patients with a previous cycle stimulated with rFSH alone showed lower fertilization rates associated with rLH supplementation. ${ }^{13}$

The use of rLH during COS is a matter of debate in the literature that has produced controversial results. In studies 
Table 2 Descriptive analysis of demographics, response to COS and laboratorial ICSI outcomes of patients $<35$ years old in repeated cycles $(n=50)$

\begin{tabular}{|c|c|c|c|}
\hline Variable & rFSH group $(n=25)$ & rFSH + rLH group $(n=25)$ & p-value \\
\hline Female age & $32.00 \pm 0.48$ & $32.05 \pm 0.54$ & 0.942 \\
\hline Male age & $36.26 \pm 1.39$ & $36.31 \pm 1.66$ & 0.981 \\
\hline BMI & $25.54 \pm 0.98$ & $24.58 \pm 1.03$ & 0.500 \\
\hline FSH dose (IU) & $2521.50 \pm 138.76$ & $2471.05 \pm 159.17$ & 0.811 \\
\hline LH dose (IU) & 0.0 & $1235.53 \pm 401.07$ & NA \\
\hline Estradiol level (pg/mL) & $1085.05 \pm 399.49$ & $1916.20 \pm 357.31$ & 0.322 \\
\hline Cycles triggered with GnRHa & $1 / 25(4.0)$ & $2 / 25(8.0)$ & 0.551 \\
\hline Follicles (n) & $12.12 \pm 1.49$ & $13.74 \pm 1.71$ & 0.475 \\
\hline Retrieved oocytes (n) & $8.12 \pm 1.09$ & $9.84 \pm 1.25$ & 0.298 \\
\hline Oocyte yield (\%) & $70.98 \pm 4.49$ & $75.54 \pm 5.16$ & 0.505 \\
\hline MII oocyte rate (\%) & $68.08 \pm 4.75$ & $67.36 \pm 5.44$ & 0.920 \\
\hline Fertilization rate (\%) & $81.08 \pm 4.47$ & $72.83 \pm 5.2$ & 0.229 \\
\hline Normal cleavage speed rate (\%) & $71.79 \pm 5.13$ & $75.32 \pm 5.97$ & 0.654 \\
\hline D3 high-quality embryos rate (\%) & $42.97 \pm 9.99$ & $40.41 \pm 12.24$ & 0.871 \\
\hline Blastocyst development rate (\%) & $41.60 \pm 12.12$ & $47.44 \pm 11.06$ & 0.859 \\
\hline Frozen embryos (n) & $1.50 \pm 0.56$ & $2.83 \pm 0.64$ & 0.118 \\
\hline Endometrial thickness (mm) & $10.30 \pm 0.59$ & $10.87 \pm 0.66$ & 0.520 \\
\hline Embryos transferred (n) & $2.33 \pm 0.14$ & $2.07 \pm 0.17$ & 0.223 \\
\hline Cycles with embryo transfer (\%) & $21 / 25(84.0)$ & $18 / 25(72.00)$ & 0.409 \\
\hline Implantation rate (\%) & $21.43 \pm 1.01$ & $38.46 \pm 1.72$ & $<0.001$ \\
\hline Pregnancy rate (\%) & $6 / 21(28.57)$ & $9 / 18(50.00)$ & 0.197 \\
\hline Miscarriage rate (\%) & $2 / 6(33.33)$ & $0 / 9(0.0)$ & 0.083 \\
\hline OHSS rate (\%) & $0 / 25(0.0)$ & $1 / 25(4.0)$ & $>0.999$ \\
\hline
\end{tabular}

Abbreviations: BMI, body mass index; COS, controlled ovarian stimulation; D3, day 3 of embryo development; ICSI, intracytoplasmic sperm injection; IU, international unit; MII, metaphase II; NA, not applicable; OHSS, ovarian hyper stimulation syndrome; rFSH, recombinant follicle stimulating hormone; rLH, recombinant luteinizing hormone.

Note: values are mean \pm standard error, unless otherwise noted.

that investigated the benefits of adding LH to FSH stimulus in women with normal response to COS, higher levels of estradiol ${ }^{14-18}$ and progesterone, ${ }^{15}$ higher rate of high-quality embryos, ${ }^{15}$ a smaller number of cycles cancelled, ${ }^{14}$ increased pregnancy rate, ${ }^{14}$ and less incidence of OHSS ${ }^{14}$ were observed compared with stimulus with rFSH alone. One study demonstrated a negative impact of LH supplementation on oocyte maturation and fertilization. ${ }^{13}$ Conversely, several studies reported no difference in the outcomes of cycles when rFSH alone was compared with $\mathrm{rFSH}+\mathrm{rLH}^{8,19-22}$

In patients with poor response to COS, stimulation with $\mathrm{rFSH}+\mathrm{rLH}$ resulted in higher pregnancy, implantation, and live birth rates when compared with stimulation with $\mathrm{rFSH}$ alone or human menopausal gonadotropin. ${ }^{23}$ Another study showed that stimulation with $\mathrm{rFSH}+\mathrm{rLH}$ yielded higher rate of high-quality embryos. ${ }^{19}$ These results suggest that poor response to COS could be related to $\mathrm{LH}$ insufficiency, and $\mathrm{rLH}$ supplementation might rescue oocyte competence that, in turn, could lead to the development of viable embryos, thus increasing pregnancy outcomes. On the other hand, some studies reported no significant differences in ICSI outcomes when comparing the two stimulation regimens in poor responder patients. ${ }^{24,25}$

Significantly increased implantation ratse $\mathrm{r}^{6,8,9}$ and live birth rates ${ }^{9}$ have been observed in older women stimulated with $\mathrm{rFSH}+\mathrm{rLH}$ when compared with their nonsupplemented counterparts. ${ }^{8}$ Moreover, treatment with rLH significantly reduced total $\mathrm{FSH}$ consumption, ${ }^{8}$ confirming that FSH and LH act synergistically. Conversely, Fábregues et al. ${ }^{26}$ showed that rLH supplementation did not increase ovarian response to COS and implantation rates in patients of older reproductive ages. Marrs et al. ${ }^{21}$ observed similar pregnancy rates in young and older women receiving $\mathrm{rFSH}+\mathrm{rLH}$; however, pregnancy rates in women $\geq 35$ years old receiving rFSH alone significantly declined when compared with those of women $<35$ years old, suggesting that these patients might benefit from the addition of rLH. It has been suggested that younger women possess a higher number of LH receptors compared with older women and, therefore, do not require LH supplementation, while LH supplementation in older women secures a sufficient LH-induced response. ${ }^{8}$ 
754 Improving Implantation Rate in 2nd ICSI Cycle through Ovarian Stimulation Setti et al.

Table 3 Descriptive analysis of demographics, response to COS and laboratorial ICSI outcomes of patients aged $\geq 35$ years old in repeated cycles in patients $(n=178)$

\begin{tabular}{|c|c|c|c|}
\hline Variable & rFSH group $(n=89)$ & rFSH + rLH group $(n=89)$ & p-value \\
\hline Female age & $38.65 \pm 0.29$ & $39.06 \pm 0.28$ & 0.303 \\
\hline Male age & $40.10 \pm 0.68$ & $40.67 \pm 0.66$ & 0.549 \\
\hline BMI & $24.70 \pm 0.47$ & $24.70 \pm 0.45$ & 0.995 \\
\hline FSH dose (IU) & $2913.69 \pm 248.86$ & $2738.16 \pm 2369.51$ & 0.611 \\
\hline LH dose (IU) & 0.0 & $1369.08 \pm 359.66$ & NA \\
\hline Estradiol level (pg/mL) & $1161.80 \pm 215.94$ & $1966.55 \pm 220.13$ & 0.009 \\
\hline Cycles triggered with $\mathrm{GnRHa}$ & $8 / 89(9.0)$ & $8 / 89(9.0)$ & $>0.999$ \\
\hline Follicles (n) & $9.39 \pm 0.77$ & $9.71 \pm 0.75$ & 0.772 \\
\hline Retrieved oocytes (n) & $5.88 \pm 0.54$ & $6.79 \pm 0.53$ & 0.227 \\
\hline Oocyte yield (\%) & $61.28 \pm 2.54$ & $68.62 \pm 2.46$ & 0.038 \\
\hline MII oocyte rate (\%) & $67.61 \pm 2.95$ & $72.33 \pm 2.83$ & 0.248 \\
\hline Fertilization rate (\%) & $76.20 \pm 2.81$ & $73.06 \pm 2.65$ & 0.417 \\
\hline Normal cleavage speed rate (\%) & $65.72 \pm 3.78$ & $72.65 \pm 3.57$ & 0.184 \\
\hline D3 high-quality embryos rate (\%) & $32.01 \pm 4.83$ & $48.81 \pm 4.69$ & 0.013 \\
\hline Blastocyst development rate (\%) & $39.06 \pm 7.34$ & $45.10 \pm 6.24$ & 0.531 \\
\hline Frozen embryos (n) & $2.42 \pm 0.75$ & $3.09 \pm 0.67$ & 0.508 \\
\hline Endometrial thickness (mm) & $10.33 \pm 0.30$ & $10.68 \pm 0.26$ & 0.386 \\
\hline Embryos transferred (n) & $1.98 \pm 0.11$ & $2.04 \pm 0.10$ & 0.712 \\
\hline Cycles with embryo transfer (\%) & 49/89 (55.06) & $51 / 89(57.30)$ & 0.698 \\
\hline Implantation rate (\%) & $17.35 \pm 0.60$ & $23.64 \pm 0.66$ & $<0.001$ \\
\hline Pregnancy rate (\%) & $9 / 49$ (18.37) & $12 / 51(23.53)$ & 0.508 \\
\hline Miscarriage rate (\%) & $3 / 9(33.33)$ & $1 / 12(8.33)$ & 0.140 \\
\hline OHSS rate (\%) & $3 / 89(3.4)$ & $5 / 89(5.6)$ & 0.469 \\
\hline
\end{tabular}

Abbreviations: BMI, body mass index; COS, controlled ovarian stimulation; D3, day 3 of embryo development; ICSI, intracytoplasmic sperm injection; IU, international unit; MII, metaphase II; NA, not applicable; OHSS, ovarian hyper stimulation syndrome; rFSH, recombinant follicle stimulating hormone; rLH, recombinant luteinizing hormone.

Note: values are mean \pm standard error, unless otherwise noted.

Table 4 Descriptive analysis of demographics, response to COS and laboratorial ICSI outcomes in repeated cycles in patients with poor response to $\operatorname{COS}$ ( $\leq 4$ retrieved oocytes) $(n=102)$

\begin{tabular}{llll}
\hline Variable & rFSH group $(\boldsymbol{n}=51)$ & rFSH + rLH group $(\boldsymbol{n}=\mathbf{5 1})$ & p-value \\
\hline Female age & $38.37 \pm 0.54$ & $38.93 \pm 0.58$ & 0.481 \\
Male age & $39.90 \pm 0.89$ & $39.97 \pm 0.98$ & 0.959 \\
BMI & $25.11 \pm 0.45$ & $24.23 \pm 0.50$ & 0.194 \\
FSH dose (IU) & $3051.60 \pm 456.34$ & $2536.05 \pm 492.09$ & 0.442 \\
LH dose (IU) & 0.0 & $1268.02 \pm 442.97$ & $\mathrm{NA}$ \\
Estradiol level (pg/mL) & $596.24 \pm 101.87$ & $725.51 \pm 111.24$ & 0.391 \\
Cycles triggered with GnRHa & $0 / 51(0.0)$ & $0 / 51(0.0)$ & $>0.999$ \\
Follicles (n) & $4.65 \pm 0.30$ & $4.33 \pm 0.33$ & 0.472 \\
Retrieved oocytes (n) & $2.29 \pm 0.16$ & $2.47 \pm 0.18$ & 0.481 \\
Oocyte yield (\%) & $56.82 \pm 1.31$ & $63.29 \pm 1.34$ & 0.001 \\
MII oocyte rate (\%) & $69.87 \pm 1.34$ & $78+12 \pm 1.56$ & $<0.001$ \\
Fertilization rate (\%) & $79.46 \pm 1.68$ & $81.0 \pm 1.8$ & 0.533 \\
Normal cleavage speed rate (\%) & $62.5 \pm 1.08$ & 75.83 & $<0.001$
\end{tabular}


Table 4 (Continued)

\begin{tabular}{llll}
\hline Variable & rFSH group $(\boldsymbol{n}=\mathbf{5 1})$ & rFSH + rLH group $(\boldsymbol{n}=\mathbf{5 1})$ & $p$-value \\
\hline D3 high-quality embryos rate (\%) & $32.47 \pm 6.44$ & $49.14 \pm 7.32$ & 0.087 \\
Blastocyst development rate (\%) & $32.81 \pm 7.23$ & $33.20 \pm 6.41$ & 0.967 \\
Frozen embryos (n) & $0.71 \pm 0.17$ & $0.69 \pm 0.19$ & 0.957 \\
Endometrial thickness (mm) & $10.22 \pm 0.45$ & $10.10 \pm 0.50$ & 0.861 \\
Embryos transferred (n) & $1.68 \pm 0.12$ & $1.68 \pm 0.13$ & 0.992 \\
Cycles with embryo transfer (\%) & $25 / 51(49.02)$ & $26 / 51(50.98)$ & 0.836 \\
Implantation rate (\%) & $10.00 \pm 0.63$ & $20.45 \pm 0.96$ & $<0.001$ \\
Pregnancy rate (\%) & $3 / 25(12.00)$ & $6 / 26(23.08)$ & 0.332 \\
Miscarriage rate (\%) & $3 / 3(100)$ & $0 / 6(0.0)$ & $<0.001$ \\
OHSS rate $(\%)$ & $0 / 51(0.0)$ & $0 / 51(0.0)$ & 0.999 \\
\hline
\end{tabular}

Abbreviations: BMI, body mass index; COS, controlled ovarian stimulation; D3, day 3 of embryo development; ICSI, intracytoplasmic sperm injection; IU, international unit; MII, metaphase II; NA, not applicable; OHSS, ovarian hyper stimulation syndrome; rFSH, recombinant follicle stimulating hormone; rLH, recombinant luteinizing hormone.

Note: values are mean \pm standard error, unless otherwise noted.

Table 5 Descriptive analysis of demographics, response to COS and laboratorial ICSI outcomes in repeated cycles in patients with normal response to $\operatorname{COS}$ ( $\geq 5$ retrieved oocytes) $(n=126)$

\begin{tabular}{|c|c|c|c|}
\hline Variable & rFSH group $(n=63)$ & rFSH + rLH group $(n=63)$ & p-value \\
\hline Female age & $36.24 \pm 0.44$ & $37.27 \pm 0.42$ & 0.092 \\
\hline Male age & $38.78 \pm 0.86$ & $39.95 \pm 0.83$ & 0.322 \\
\hline $\mathrm{BMI}$ & $24.68 \pm 0.66$ & $24.94 \pm 0.60$ & 0.770 \\
\hline FSH dose (IU) & $2648.61 \pm 75.42$ & $2789.08 \pm 71.04$ & 0.175 \\
\hline LH dose (IU) & 0.0 & $1394.54 \pm 308.62$ & NA \\
\hline Estradiol level (pg/mL) & $1725.74 \pm 303.65$ & $2788.37 \pm 281.12$ & 0.010 \\
\hline Cycles triggered with GnRHa & $9 / 63(14.3)$ & $10 / 63$ (15.9) & 0.803 \\
\hline Follicles (n) & $14.32 \pm 0.91$ & $14.04 \pm 0.86$ & 0.826 \\
\hline Retrieved oocytes (n) & $9.67 \pm 0.61$ & $10.23 \pm 0.57$ & 0.503 \\
\hline Oocyte yield (\%) & $75.37 \pm 1.99$ & $82.69 \pm 1.78$ & 0.006 \\
\hline MII oocyte rate (\%) & $73.33 \pm 1.43$ & $71.8 \pm 1.69$ & 0.489 \\
\hline Fertilization rate (\%) & $78.31 \pm 2.65$ & $72.40 \pm 2.51$ & 0.105 \\
\hline Normal cleavage speed rate (\%) & $70.02 \pm 3.16$ & $72.54 \pm 3.00$ & 0.565 \\
\hline D3 high-quality embryos rate (\%) & $35.80 \pm 5.96$ & $46.78 \pm 5.45$ & 0.174 \\
\hline Blastocyst development rate (\%) & $41.79 \pm 11.63$ & $53.29 \pm 9.59$ & 0.445 \\
\hline Frozen embryos (n) & $3.71 \pm 0.99$ & $4.23 \pm 0.80$ & 0.680 \\
\hline Endometrial thickness (mm) & $10.40 \pm 0.33$ & $10.98 \pm 0.27$ & 0.182 \\
\hline Embryos transferred (n) & $2.29 \pm 0.107$ & $2.21 \pm 108$ & 0.603 \\
\hline Cycles with embryo transfer (\%) & $45 / 63(71.43)$ & $42 / 63(66.67)$ & 0.513 \\
\hline Implantation rate (\%) & $23.33 \pm 0.72$ & $29.35 \pm 0.80$ & $<0.001$ \\
\hline Pregnancy rate (\%) & $12 / 43(27.91)$ & $13 / 42(30.95)$ & 0.579 \\
\hline Miscarriage rate (\%) & $2 / 12(16.67)$ & $1 / 13(7.69)$ & 0.425 \\
\hline OHSS rate (\%) & $3 / 63(4.76)$ & $6 / 63(9.52)$ & 0.299 \\
\hline
\end{tabular}

Abbreviations: BMI, body mass index; COS, controlled ovarian stimulation; D3, day 3 of embryo development; ICSI, intracytoplasmic sperm injection; IU, international unit; MII, metaphase II; NA, not applicable; OHSS, ovarian hyper stimulation syndrome; rFSH, recombinant follicle stimulating hormone; rLH, recombinant luteinizing hormone.

Note: values are mean \pm standard error, unless otherwise noted. 
Moreover, ovarian androgen secretion is also diminished in older women, suggesting an age-related decline in ovarian response to stimulation with $\mathrm{LH}^{8}$

Few studies investigated the potential benefits of adding rLH to rFSH in patients with reduced serum LH concentrations. Lisi et al. ${ }^{27}$ showed an increased implantation rate in women with $\mathrm{LH}$ concentration $<1.0 \mathrm{IU} / \mathrm{l}$ at downregulation who received rLH supplementation, suggesting that, in the experience of profound LH downregulation, rLH supplementation might be beneficial. In opposition, Humaidan et al. ${ }^{8}$ observed increased implantation rates when LH supplementation was used in patients with endogenous LH concentrations $\geq 1.99 \mathrm{IU} / \mathrm{l}$. One study failed to demonstrate association between rLH supplementation and improved outcomes. $^{28}$

The lack of consensus in the aforementioned literature has led to the publication of several meta-analyses, which also came to conflicting conclusions. The meta-analyses suggested that $\mathrm{rFSH}+\mathrm{rLH}$ results in shorter stimulation length and fewer rFSH consumption, ${ }^{29}$ and yields higher estradiol levels ${ }^{29,30}$ and higher number of mature oocytes. ${ }^{30}$ While several metaanalysis showed no significant differences in implantation, 2,29,30 pregnancy, $^{2,29,30}$ and live-birth rates, 2,11,30,31 others have demonstrated higher implantation rates, ${ }^{3}$ pregnancy rates, ${ }^{3,32}$ ongoing pregnancy rates, ${ }^{11}$ and lower miscarriage rates ${ }^{11}$ in the recombinant $\mathrm{LH}$-supplemented regimen.

For poor responder patients, an increase in clinical pregnancy rate was observed in favor of supplementing rLH. ${ }^{2,32}$ In addition, poor responders showed significantly more retrieved oocytes with rFSH + rLH compared with rFSH alone. ${ }^{32}$

The disparity found in the literature may be due to (i) $\mathrm{LH}$ administration start (beginning of treatment or late phase), (ii) type of GnRH analogue used (agonist or antagonist), (iii) starting dose of gonadotropin and gonadotropin dose adjustment during COS, (iv) heterogeneous definition of poor ovarian response, and (v) heterogeneous cutoff values for advanced maternal age ( 35 or 36 years old).

The possible mechanisms behind the benefits offered by rLH supplementation are improved oocyte competence and endometrial receptivity. Lower levels of cumulous cell apoptosis have been demonstrated in cycles with rLH supplementation as compared with cycles with rFSH alone, ${ }^{33}$ which can reflect enhanced oocyte competence. In addition, $\mathrm{LH}$ stimulates CYP17 to convert progesterone into androgens, which in turn can be aromatized to estrogens. The supplementation with $\mathrm{LH}$ decreases the chance of a premature progesterone rise prior to luteinization, thus benefitting the endometrium and increasing the chance of implantation and clinical pregnancy. ${ }^{34}$ Finally, the addition of rLH may improve follicular insulin sensitivity, leading to decreased androgen levels through a cascade mediated by increased production of adiponectin. This favorable setting may culminate in enhanced follicular maturation, ovulation, and fertilization capacity. ${ }^{35}$

This is a retrospective study with its inherent limitations and bias. In addition, although the sample size was adequate for the analysis of the general group, the present study is underpowered for subgroups analyses. The present study was limited by its small sample size but creates a rationale to conduct randomized studies with larger casuistic to draw concrete conclusions about the use of rFSH and rLH for ovarian stimulation in patients with cycles stimulated with rFSH alone. The results presented here might provide another tool for the clinician to use in the decision-making process regarding the trigger regimen. The most important take home message is that the outcomes of ICSI cycles from unselected patients can be improved in a following cycle with the use of LH supplementation for ovarian stimulation.

\section{Conclusion}

In conclusion, ovarian stimulation with LH supplementation results in higher implantation rates, regardless of maternal age and response to COS, compared with cycles stimulated with rFSH only. Improvements were also observed for ICSI laboratory outcomes and miscarriage rate when the patients were stratified by age and number of retrieved oocytes. Despite being encouraging, due to the retrospective nature of the present study, these results should be confirmed in randomized controlled trials.

\section{Conflict of Interests}

The authors have no conflict of interests to declare.

\section{References}

1 Raju GA, Chavan R, Deenadayal M, Gunasheela D, Gutgutia R, Haripriya $G$, et al. Luteinizing hormone and follicle stimulating hormone synergy: A review of role in controlled ovarian hyperstimulation. J Hum Reprod Sci. 2013;6(04):227-234. Doi: 10.4103/0974-1208.126285

2 Mochtar MH, Van der Veen F, Ziech M, van Wely M. Recombinant Luteinizing Hormone (rLH) for controlled ovarian hyperstimulation in assisted reproductive cycles. Cochrane Database Syst Rev. 2007;(02):CD005070. Doi: 10.1002/14651858.CD005070.pub2

3 Hill MJ, Levens ED, Levy G, Ryan ME, Csokmay JM, DeCherney AH, et al. The use of recombinant luteinizing hormone in patients undergoing assisted reproductive techniques with advanced reproductive age: a systematic review and meta-analysis. Fertil Steril. 2012;97(05):1108-14.e1. Doi: 10.1016/j.fertnstert.2012. 01.130

4 Alviggi C, Clarizia R, Mollo A, Ranieri A, De Placido G. Who needs LH in ovarian stimulation? Reprod Biomed Online. 2011;22 (Suppl 1):S33-S41. Doi: 10.1016/S1472-6483(11)60007-2

5 Alviggi C, Humaidan P, Ezcurra D. Hormonal, functional and genetic biomarkers in controlled ovarian stimulation: tools for matching patients and protocols. Reprod Biol Endocrinol. 2012; 10:9. Doi: 10.1186/1477-7827-10-9

6 Bosch E, Labarta E, Crespo J, Simón C, Remohí J, Pellicer A. Impact of luteinizing hormone administration on gonadotropin-releasing hormone antagonist cycles: an age-adjusted analysis. Fertil Steril. 2011;95(03):1031-1036. Doi: 10.1016/j.fertnstert.2010. 10.021

7 Hill MJ, Levy G, Levens ED. Does exogenous LH in ovarian stimulation improve assisted reproduction success? An appraisal of the literature. Reprod Biomed Online. 2012;24(03):261-271. Doi: 10.1016/j.rbmo.2011.12.005

8 Humaidan P, Bungum M, Bungum L, Yding Andersen C. Effects of recombinant $\mathrm{LH}$ supplementation in women undergoing assisted reproduction with $\mathrm{GnRH}$ agonist down-regulation and 
stimulation with recombinant FSH: an opening study. Reprod Biomed Online. 2004;8(06):635-643. Doi: 10.1016/s1472-6483 (10)61643-4

9 Matorras R, Prieto B, Exposito A, Mendoza R, Crisol L, Herranz P, et al. Mid-follicular LH supplementation in women aged 35-39 years undergoing ICSI cycles: a randomized controlled study. Reprod Biomed Online. 2009;19(06):879-887. Doi: 10.1016/j. rbmo.2009.09.016

10 Wong PC, Qiao J, Ho C, Ramaraju GA, Wiweko B, Takehara Y, et al; Asia Pacific Fertility Advisory Group. Current opinion on use of luteinizing hormone supplementation in assisted reproduction therapy: an Asian perspective. Reprod Biomed Online. 2011;23 (01):81-90. Doi: 10.1016/j.rbmo.2011.03.023

11 Mochtar MH, Danhof NA, Ayeleke RO, Van der Veen F, van Wely M. Recombinant luteinizing hormone (rLH) and recombinant follicle stimulating hormone (rFSH) for ovarian stimulation in IVF/ICSI cycles. Cochrane Database Syst Rev. 2017;5(05):CD005070. Doi: 10.1002/14651858.CD005070.pub3

12 Palermo G, Joris H, Devroey P, Van Steirteghem AC. Pregnancies after intracytoplasmic injection of single spermatozoon into an oocyte. Lancet. 1992;340(8810):17-18. Doi: 10.1016/0140-6736 (92)92425-f

13 Balasch J, Creus M, Fábregues F, Carmona F, Puerto B, Casamitjana $\mathrm{R}$, et al. The effect of exogenous luteinizing hormone ( $\mathrm{LH})$ on oocyte viability: evidence from a comparative study using recombinant human follicle-stimulating hormone (FSH) alone or in combination with recombinant $\mathrm{LH}$ for ovarian stimulation in pituitary-suppressed women undergoing assisted reproduction. J Assist Reprod Genet. 2001;18(05):250-256. Doi: 10.1023/ a:1016662100572

14 Caserta D, Lisi F, Marci R, Ciardo F, Fazi A, Lisi R, et al. Does supplementation with recombinant luteinizing hormone prevent ovarian hyperstimulation syndrome in down regulated patients undergoing recombinant follicle stimulating hormone multiple follicular stimulation for IVF/ET and reduces cancellation rate for high risk of hyperstimulation? Gynecol Endocrinol. 2011;27(11): 862-866. Doi: 10.3109/09513590.2010.544133

15 Wiser A, Hourvitz A, Yinon Y, Levron J, Dor J, Elizur S. Recombinant human luteinizing hormone supplementation may improve embryo quality in in vitro fertilization/intracytoplasmic sperm injection cycles with gonadotropin-releasing hormone antagonist protocol. Open J Obstet Gynecol. 2011;1(02):31-35. Doi: 10.4236/ojog.2011.12007

16 Cédrin-Durnerin I, Grange-Dujardin D, Laffy A, Parneix I, Massin $\mathrm{N}$, Galey J, et al. Recombinant human LH supplementation during GnRH antagonist administration in IVF/ICSI cycles: a prospective randomized study. Hum Reprod. 2004;19(09):1979-1984. Doi: 10.1093/humrep/deh369

17 Griesinger G, Schultze-Mosgau A, Dafopoulos K, Schroeder A, Schroer A, von Otte S, et al. Recombinant luteinizing hormone supplementation to recombinant follicle-stimulating hormone induced ovarian hyperstimulation in the GnRH-antagonist multiple-dose protocol. Hum Reprod. 2005;20(05):1200-1206. Doi: 10.1093/humrep/deh741

18 Levi-Setti PE, Cavagna M, Bulletti C. Recombinant gonadotrophins associated with GnRH antagonist (cetrorelix) in ovarian stimulation for ICSI: comparison of r-FSH alone and in combination with r-LH. Eur J Obstet Gynecol Reprod Biol. 2006;126(02):212-216. Doi: 10.1016/j.ejogrb.2005.11.023

19 Musters AM, van Wely M, Mastenbroek S, Kaajk EM, Repping S, van der Veen $\mathrm{F}$, et al. The effect of recombinant $\mathrm{LH}$ on embryo quality: a randomized controlled trial in women with poor ovarian reserve. Hum Reprod. 2012;27(01):244-250. Doi: 10.1093/humrep/der371

20 NyboeAndersen A, Humaidan P, Fried G, Hausken J, Antila L, Banhsbøll , et al; Nordic LH study group. Recombinant LH supplementation to recombinant FSH during the final days of controlled ovarian stimulation for in vitro fertilization. A multicentre, prospective, randomized, controlled trial. Hum Reprod. 2008;23 (02):427-434. Doi: 10.1093/humrep/dem317

21 Marrs R, Meldrum D, Muasher S, Schoolcraft W, Werlin L, Kelly E. Randomized trial to compare the effect of recombinant human FSH (follitropin alfa) with or without recombinant human LH in women undergoing assisted reproduction treatment. Reprod Biomed Online. 2004;8(02):175-182. Doi: 10.1016/s1472-6483 (10)60513-5

22 Berkkanoglu M, Isikoglu M, Aydin D, Ozgur K. Clinical effects of ovulation induction with recombinant follicle-stimulating hormone supplemented with recombinant luteinizing hormone or low-dose recombinant human chorionic gonadotropin in the midfollicular phase in microdose cycles in poor responders. Fertil Steril. 2007;88(03):665-669. Doi: 10.1016/j.fertnstert.2006.11.150

23 Ferraretti AP, Gianaroli L, Magli MC, D'angelo A, Farfalli V, Montanaro N. Exogenous luteinizing hormone in controlled ovarian hyperstimulation for assisted reproduction techniques. Fertil Steril. 2004;82(06):1521-1526. Doi: 10.1016/j.fertnstert.2004.06.041

24 Motta E, Massaguer A, Serafini P, Beltrame A, Yadid I, Coslowsky M. Supplementation with rec-FSH or rec-LH is equally effective to modulate sub-optimal response for IVF cycles. A prospective randomized trial. Hum Reprod. 2005;20(Suppl 1):i125

25 Barrenetxea G, Agirregoikoa JA, Jiménez MR, de Larruzea AL, Ganzabal T, Carbonero K. Ovarian response and pregnancy outcome in poor-responder women: a randomized controlled trial on the effect of luteinizing hormone supplementation on in vitro fertilization cycles. Fertil Steril. 2008;89(03):546-553. Doi: 10.1016/j.fertnstert.2007.03.088

26 Fábregues F, Creus M, Peñarrubia J, Manau D, Vanrell JA, Balasch J. Effects of recombinant human luteinizing hormone supplementation on ovarian stimulation and the implantation rate in downregulated women of advanced reproductive age. Fertil Steril. 2006;85(04):925-931. Doi: 10.1016/j.fertnstert.2005.09.049

27 Lisi F, Rinaldi L, Fishel S, Pepe GP, Picconeri MG, Campbell A, et al. Use of recombinant LH in a group of unselected IVF patients. Reprod Biomed Online. 2002;5(02):104-108. Doi: 10.1016/ s1472-6483(10)61610-0

28 Lahoud R, Ryan J, Illingworth P, Quinn F, Costello M. Recombinant LH supplementation in patients with a relative reduction in $\mathrm{LH}$ levels during IVF/ICSI cycles: A prospective randomized controlled trial. Eur J Obstet Gynecol Reprod Biol. 2017; 210:300-305. Doi: 10.1016/j.ejogrb.2017.01.011

29 Oliveira JB, Mauri AL, Petersen CG, Martins AN, Cornicelli J, Cavanha M, et al. Recombinant luteinizing hormone supplementation to recombinant follicle-stimulation hormone during induced ovarian stimulation in the GnRH-agonist protocol: a meta-analysis. J Assist Reprod Genet. 2007;24(2-3):67-75. Doi: 10.1007/s10815-006-9095-4

30 Baruffi RL, Mauri AL, Petersen CG, Felipe V, Mc Martins A, Cornicelli J, et al. Recombinant LH supplementation to recombinant FSH during induced ovarian stimulation in the GnRHantagonist protocol: a meta-analysis. Reprod Biomed Online. 2007;14(01):14-25. Doi: 10.1016/s1472-6483(10)60758-4

31 Kolibianakis EM, Kalogeropoulou L, Griesinger G, Papanikolaou EG, Papadimas J, Bontis J, et al. Among patients treated with FSH and GnRH analogues for in vitro fertilization, is the addition of recombinant LH associated with the probability of live birth? A systematic review and meta-analysis. Hum Reprod Update. 2007; 13(05):445-452. Doi: 10.1093/humupd/dmm008

32 Lehert P, Kolibianakis EM, Venetis CA, Schertz J, Saunders H, Arriagada $\mathrm{P}$, et al. Recombinant human follicle-stimulating hormone ( $\mathrm{r}-\mathrm{hFSH})$ plus recombinant luteinizing hormone versus r-hFSH alone for ovarian stimulation during assisted reproductive technology: systematic review and meta-analysis. Reprod Biol Endocrinol. 2014;12(01):17. Doi: 10.1186/1477$7827-12-17$ 
758 Improving Implantation Rate in 2nd ICSI Cycle through Ovarian Stimulation Setti et al.

33 Ruvolo G, Bosco L, Pane A, Morici G, Cittadini E, Roccheri MC. Lower apoptosis rate in human cumulus cells after administration of recombinant luteinizing hormone to women undergoing ovarian stimulation for in vitro fertilization procedures. Fertil Steril. 2007;87(03):542-546. Doi: 10.1016/j.fertnstert.2006.06.059

34 Hill MJ, Propst AM. The use of rLH, HMG and hCG in controlled ovarian stimulation for assisted reproductive technologies. In:
Darwish A, ed. Enhancing success of assisted reproduction. Rijeka: InTechOpen; 2012:53-76

35 Gutman G, Barak V, Maslovitz S, Amit A, Lessing JB, Geva E. Recombinant luteinizing hormone induces increased production of ovarian follicular adiponectin in vivo: implications for enhanced insulin sensitivity. Fertil Steril. 2009;91(05):1837-1841. Doi: 10.1016/j.fertnstert.2008.02.006 